\title{
Reconstruction of Radiation Dose Received by Diagnostic Radiologic Technologists in Korea
}

\author{
Yeongchull Choi', Jaeyoung Kim ${ }^{1}$, Jung Jeung Lee', Jae Kwan Jun², Won Jin Lee ${ }^{3}$ \\ ${ }^{1}$ Department of Preventive Medicine, Keimyung University School of Medicine, Daegu; ${ }^{2}$ National Cancer Control Institute, National Cancer Center, \\ Goyang; ${ }^{3}$ Department of Preventive Medicine, Korea University College of Medicine, Seoul, Korea
}

Objectives: Diagnostic medical radiation workers in Korea have been officially monitored for their occupational radiation doses since 1996. The purpose of this study was to design models for reconstructing unknown individual radiation doses to which diagnostic radiation technologists were exposed before 1996.

Methods: Radiation dose reconstruction models were developed by using cross-sectional survey data and the personal badge doses of 8167 radiologic technologists. The models included calendar year and age as predictors, and the participants were grouped into six categories according to their sex and facility type. The annual doses between 1971 and 1995 for those who were employed before 1996 were estimated using these models.

Results: The calendar year and age were inversely related to the estimated radiation doses in the models of all six groups. The annual median estimated doses decreased from $9.45 \mathrm{mSv}$ in 1971 to $1.26 \mathrm{mSv}$ in 1995, and the associated dose variation also decreased with time. The estimated median badge doses from 1996 (1.22 mSv) to 2011 (0.30 mSv) were similar to the measured doses (1.68 mSv to $0.21 \mathrm{mSv}$ ) for the same years. Similar results were observed for all six groups.

Conclusions: The reconstruction models developed in this study may be useful for estimating historical occupational radiation doses received by medical radiologic technologists in Korea.

Key words: Dosimetry, Historical reconstruction, Occupational exposure, Radiation, Workers

\section{INTRODUCTION}

Exposure information is essential for radiation epidemiologic research that aims to determine of the various health consequences of radiation. Because occupational radiation exposure

Received: June 28, 2016 Accepted: August 18, 2016

Corresponding author: Won Jin Lee, MD, PhD

73 Inchon-ro, Seongbuk-gu, Seoul 02841, Korea.

Tel: +82-2-2286-1413, Fax: +82-2-927-7220

E-mail: leewj@korea.ac.kr

This is an Open Access article distributed under the terms of the Creative Commons Attribution Non-Commercial License (http://creativecommons.org/licenses/by$\mathrm{nc} / 4.0 /$ ) which permits unrestricted non-commercial use, distribution, and reproduction in any medium, provided the original work is properly cited. is protracted and cumulative rather than incidental, individual dose data throughout entire employment periods are needed for determining the dose-response relationship with health effects [1]. However, complete information about exposure to radiation is seldom available because many individual exposures, particularly those that occurred in the remote past, remain undocumented. One way to overcome the problem of insufficient exposure data is to reconstruct the undocumented past doses by using the known doses and the related variables that determine exposure levels, and to assign these values to individuals [1].

Several studies have addressed historical dose reconstruction for external radiation exposure using different occupational exposure settings. Boice et al. [2] reported a reconstruc- 
tion method and results for 5801 workers who participated in research at a nuclear reactor during the period from 1948 to 1999. Bouville et al. [3] also performed dose reconstruction for the Million Worker Study in the US Eheman and Tolbert [4] developed a job exposure matrix for a wide range of occupations, including military service members, nuclear fuel cycle workers, NASA employees, and medical workers, and provided the estimated annual dose distributions, categorized in terms of time.

Several studies specifically focused on radiologic technologists in different regions. Yoshinaga et al. [5] reported a dose reconstruction model that utilized information on the work history of 3461 diagnostic radiologists in Japan. While their results demonstrated a decreasing trend in the annual dose, the reconstructed values were highly variable and systematically overestimated. Simon et al. [6,7] reconstructed occupational radiation doses for 90000 US radiologic technologists for the period 1916 to 1984. Using three models for different time periods, they found a more than 40 -fold reduction in the mean annual badge dose, from $100 \mathrm{mSv}$ before 1940 to $2.3 \mathrm{mSv}$ during the 1977 to 1984 period. Zhang et al. [8] reconstructed past doses by using survey information on different types of past working conditions obtained from 3545 cohort participants, and simulated workloads associated with the working conditions of Chinese diagnostic X-ray workers. They presented the results in the form of average annual doses for 5-year-long periods, from 1950 to 1994, and demonstrated that the downward slope was steeper for the earlier periods.

A cohort study of diagnostic radiation workers was launched in Korea in 2011 [9]. As of May 2013, 12387 radiologic technologists were enrolled in the cohort who accounted for $63 \%$ of the eligible population, and completed the baseline survey. Then, the survey data were matched to the individual badge readings, the central cancer registry, and the national health insurance data. The incompleteness of exposure information, however, remains one of the obstacles for conducting epidemiologic investigations, because the dose registry does not include badge measurements before 1996. Considering the downward trend in the occupational radiation exposure, which implies higher levels of exposure in the past [10], incomplete dose information could lead to insufficient information on cumulative exposure.

This study, therefore, aimed to design models for reconstructing unknown individual radiation doses received by diagnostic radiation technologists before 1996. By reconstructing the past doses, we expect this study to help determine cu- mulative radiation exposures, which subsequently are likely to contribute to identifying the dose-response relationships pertaining to occupational radiation exposure.

\section{METHODS}

\section{Study Participants and Data Selection}

The study population included 11265 radiologic technologists who had dosimetry data in a diagnostic radiation workers' health survey in Korea [9]. To filter out improperly measured badge doses, only those who responded "I always wear a badge" and "I place my badge inside a lead apron" were chosen. Next, we also excluded those individuals for whom information on the type of facility where they were working was not available. At the end of this selection process, 8189 participants, along with their badge doses, were selected as eligible participants for our models. Informed written consent, including permission to access radiation dosimetry data, was voluntarily obtained from all individual study participants prior to enrollment. This study was reviewed and approved by the institutional review board of Korea University (KU-IRB-12-12-A-1).

The selected data were transformed into a long type, in which multiple dose measurements for the same person during separate years were recorded as unique observations in separate rows. Data containing 69413 badge doses were ob-

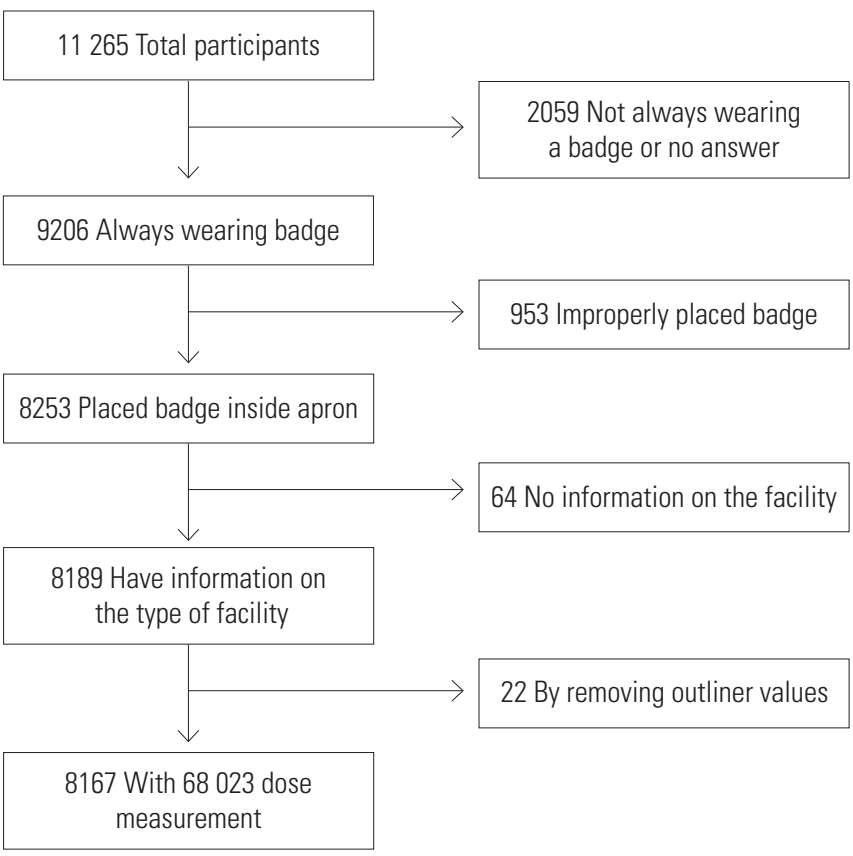

Figure 1. Selection process, manipulation of data, and the resulting number of participants or badge doses in each step. 
tained by this reshaping. Outliers above three standard deviations of the log dose for each year were excluded from the model dataset, which resulted in the removal of the data of 22 participants from the dataset. Consequently, 68023 dose measurements for 8167 radiologic technologists were retained in the final dataset that was used for the modeling. Figure 1 shows the selection and manipulation processes and the corresponding number of eligible participants.

\section{Information Used}

Two information sources were used for the modeling: a cross-sectional survey of diagnostic radiation workers and the national dose registry. The survey collected the information on the types and frequencies of radiologic tasks performed by these individuals, safety conditions and personal protection behavior, badge-wearing habits, types of facilities, and general demographic characteristics. Individual badge dose data from 1996 to 2011 were acquired from the national dose registry. All radiologic technologists are required to wear badge dosimeters in the workplace according to the 1994 amendment of the Medical Service Act [11]. Since medical facilities operating radiologic equipment are mandated to report their radiationexposed workers' badge doses every quarter to the Korea Food and Drug Administration, the registry retains a complete set of badge dose measurements. These data were matched to the survey information by using individual identifiers, and integrated into a single dataset. Quarterly doses were cumulated into yearly doses for each individual participant. These data, which contained both the information from the survey and that from the annual dose measurements, were used as the datasets for developing our dose reconstruction model.

\section{Construction of Model}

We developed a dose reconstruction model by adopting the findings of previous studies conducted in Japan [5] and China [8], in which past unknown doses were estimated as a function of time. We modified the model by adding age at the year of exposure as an additional predictor variable (Equation 1). The model features a log-linear function to account for the log-normal distribution of individual annual badge doses. The time variable corresponds to the number of years since 1970 . Using 1970 as the first year is justified by considering that only 27 out of the 8167 participants began their radiation-exposed work before 1970.

$$
\log (\text { annual dose })=\beta_{0}+\beta_{1}\left(\text { year-1970) }+\beta_{2}\right. \text { (age) ---- Equation (1) }
$$

A tree regression analysis was conducted to identify the primary predictors determining the measured badge doses, as shown in Supplemental Figures 1 and 2. Sex and age at the year of exposure were identified as the two principal determinants of exposure. Since age at the year of exposure was inversely proportional to the exposure dose, this variable was incorporated into the model rather than being used as a stratifying variable.

The participants were grouped into six categories, according to their sex and the type of facility where they worked. Three types of facilities were considered: 1) "Hospital," which included tertiary and secondary hospitals; 2) "Clinic"; and 3) "Others," which included dental clinics and hospitals, and community health centers. Next, the parameters of the model were estimated for each of the six groups to yield six reconstruction models. The number of participants and their annual badge doses in each of the six groups are listed in Table 1. Group 1 (male radiologic technologists in the "hospital" category) comprised the largest proportion of participants and doses, while group 6 (females in the "others" category) comprised the smallest one (Table 1).

Next, for each of the six groups, we estimated three parameters for the two predictors and the constant term in Equation (1): $\beta_{0}$ for the constant term, $\beta_{1}$ for the number of years since 1970 , and $\beta_{2}$ for the age at the year of exposure. Using these models, we estimated the annual doses between 1971 and 1995 for those who were exposed to radiation before 1996. The individual annual doses between 1996 and 2011 were also estimated using these models and compared with the measured badge doses for the same year. The agreement between the estimated and the measured badge doses was examined using the intraclass correlation coefficient.

For the purpose of model selection, we also compared the

Table 1. Combinations of sex and type of facility for 6 groups, and their corresponding number of participants and badge doses for radiologic technologists

\begin{tabular}{lllc}
\hline Group & Sex & \multicolumn{1}{c}{ Facility type } & $\begin{array}{c}\text { No. of } \\
\text { persons }\end{array}$ \\
\hline 1 & Male & General hospital/hospital & 3874 \\
2 & Male & Clinic & 1385 \\
3 & Male & Dental/community health center/others & 349 \\
4 & Female & General hospital/hospital & 1319 \\
5 & Female & Clinic & 992 \\
6 & Female & Dental/community health center/others & 248 \\
Total & & & 8167 \\
\hline
\end{tabular}


performance of three different models including different combinations of predictor variables. The first model used a single predictor and the year of exposure. The second one added the age in the year of exposure to the first one. The third one included additional information on the types and frequencies of the radiologic tasks of individuals. Then, 10 -fold cross-validations were performed to compare the test mean squared errors among these models. While the second model showed improvement as compared to the first, the most complex model revealed varying results across different groups when compared with the results from the second model. Combining these findings and considering the lack of information on the past work history of individual participants, we decided that it was reasonable to choose the second model as the optimal model for the reconstruction.

\section{RESULTS}

For the 8167 radiologic technologists included in the model's dataset, the ratio of males to females was 2:1, and approximately $40 \%$ were in their thirties. Nearly two-thirds were working at either tertiary or secondary hospitals, while $29.1 \%$ were working at clinics. The number of those working in metropolitan regions was larger (54.1\%) than the number of those working in non-metropolitan areas (45.9\%). More than half of the participants began their career as radiologic technologists after 2000, while those who began in 1970 or before consti- tuted only $0.4 \%$ of the enrollees (Table 2 ).

Annual median badge doses for each group are shown in

Table 2. Selected characteristics of radiologic technologists included in the study

\begin{tabular}{lrr}
\hline Characteristics & $\mathbf{n}^{\mathbf{1}}$ & $\%$ \\
\hline Sex & 5608 & 68.7 \\
$\quad$ Male & 2559 & 31.3 \\
Female & & \\
Age in the year of survey (y) & 1856 & 22.7 \\
23-29 & 3426 & 42.0 \\
30-39 & 2117 & 25.9 \\
40-49 & 691 & 8.5 \\
50-59 & 77 & 0.9 \\
$\geq 60$ & & \\
Type 0f facility & 5193 & 63.6 \\
Hospital & 2377 & 29.1 \\
Clinic & 597 & 7.3 \\
Others & & \\
Region of facility & 4095 & 54.1 \\
Metropolitan & 3473 & 45.9 \\
Non-metropolitan & & \\
Work start year & 34 & 0.4 \\
1970 or before & 155 & 1.9 \\
1971-1980 & 1089 & 26.9 \\
1981-1990 & 2189 & \\
1991-2000 & 4664 & \\
2001 and after & & \\
\hline
\end{tabular}

${ }^{1}$ Numbers may not sum up to the total because of certain missing information.

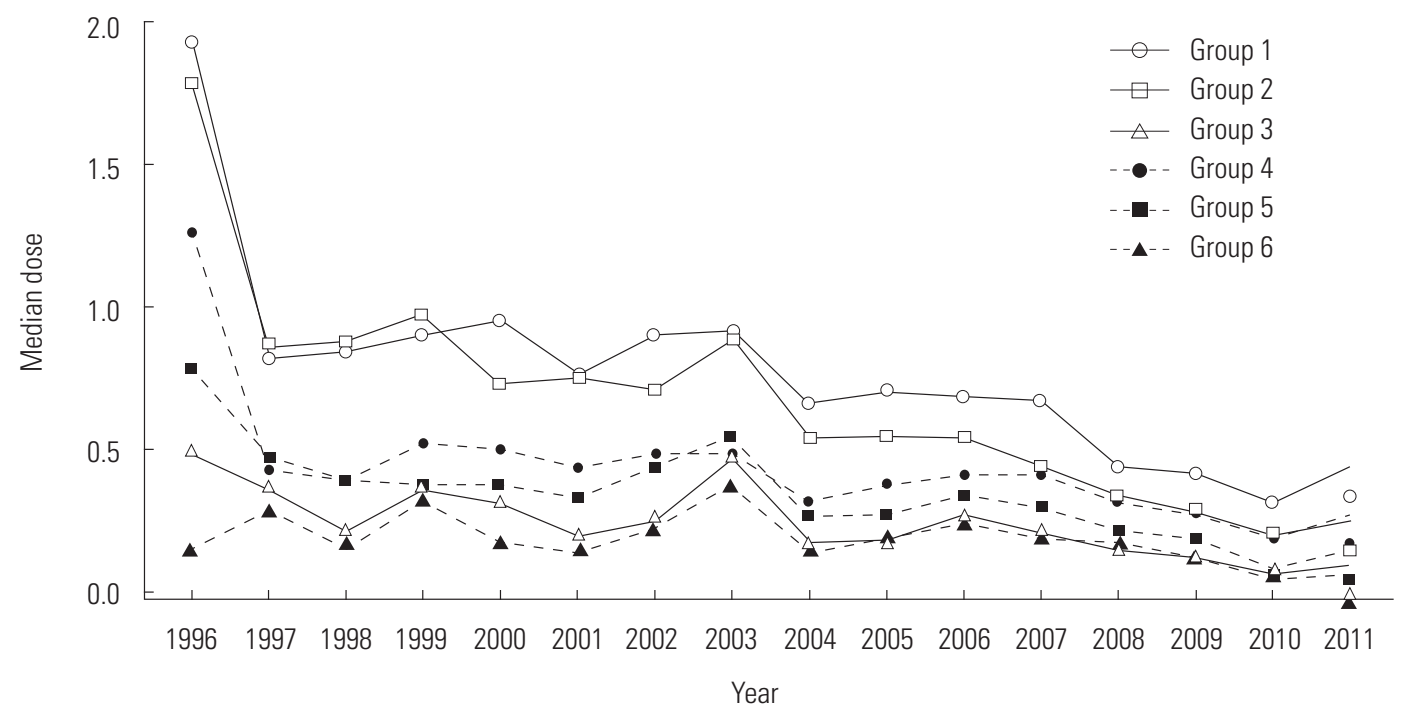

Figure 2. Annual median badge doses received by radiologic technologists categorized by group, 1996-2011. Group 1, male hospital workers; Group 2, male clinic workers; Group 3, male others workers; Group 4, female hospital workers; Group 5, female clinic workers; Group 6, female others workers. 
Figure 2. Downward trends were observed for all six groups, although the declining patterns were less clear for some groups. Relatively significant drops between 1996 and 1997 were noticed for groups 1, 2, 4, and 5. Group 1 (male hospital workers) exhibited the highest median doses for most of the years, followed by group 2 (male clinic workers).

Table 3 lists the parameters of the model predictor variables. For all groups, both $\beta_{1}$ and $\beta_{2}$ were negative, indicating that these two variables are inversely related to the estimated log doses in the models of all six groups. All of the 18 estimated parameters were statistically significant at the 0.05 significance level. The parameters for the years since $1970\left(\beta_{1}\right)$ ranged from -0.087 for group 2 to -0.042 for group 4 , while those for age at the exposure year $\left(\beta_{2}\right)$ ranged from -0.058 for group 3 to -0.015 for group 5 .

The annual median estimated doses and the comparison with the annual median badge readings from 1996 to 2011 are shown in Figure 3. For all of the participants, the median

Table 3. Parameters of the explanatory variables in the model for each group of radiologic technologists

\begin{tabular}{llllll}
\hline Group & Sex & $\begin{array}{c}\text { Type of } \\
\text { facility }\end{array}$ & $\boldsymbol{B}{ }^{1}$ & $\boldsymbol{B}^{1}{ }^{1}$ & $\boldsymbol{B}{ }^{1}$ \\
\hline 1 & Male & Hospital & 3.316 & -0.062 & -0.047 \\
2 & Male & Clinic & 3.345 & -0.087 & -0.028 \\
3 & Male & Others & 2.701 & -0.061 & -0.058 \\
4 & Female & Hospital & 1.433 & -0.042 & -0.035 \\
5 & Female & Clinic & 1.943 & -0.080 & -0.015 \\
6 & Female & Others & 2.051 & -0.063 & -0.052 \\
\hline
\end{tabular}

${ }^{1} p$-values for all parameters were less than 0.001 . estimated doses decreased from $1.22 \mathrm{mSv}$ in 1996 to $0.30 \mathrm{mSv}$ in 2011, while the measured doses ranged from $1.68 \mathrm{mSv}$ in 1996 to $0.21 \mathrm{mSv}$ in 2010. Similar results were obtained for all six groups (Supplemental Figures 3 and 4). The mean intraclass correlation coefficient between the estimated and the measured log average annual dose was 0.40 .

Figure 4 and Supplemental Table 1 show the distributions of the reconstructed annual doses received by those who worked between 1971 and 1995, that is, the period for which no measured badge dose data are available. The median estimated doses decreased from $9.45 \mathrm{mSv}$ in 1971 to $1.26 \mathrm{mSv}$ in 1995. The reconstructed dose range also narrowed, from $7.77 \mathrm{mSv}$ (minimal dose of $4.04 \mathrm{mSv}$, maximal dose of $11.81 \mathrm{mSv}$ ) in 1971 to $1.81 \mathrm{mSv}$ (minimal dose of $0.07 \mathrm{mSv}$, maximal dose of $1.87 \mathrm{mSv}$ ) in 1995. More rapid inter-annual changes in the more remote past were found.

\section{DISCUSSION}

Our reconstructed occupational radiation doses exhibited downward temporal trends, and the results were consistent for all study groups. Because the measured and the estimated median radiation doses for the 1996 to 2011 period agreed fairly well, our model may be useful for estimating historical occupational radiation doses received by medical radiation workers in Korea. However, the models should be open to further improvement by incorporating detailed past information, which is not currently considered in the model.

The downward temporal trends for the annual badge doses,

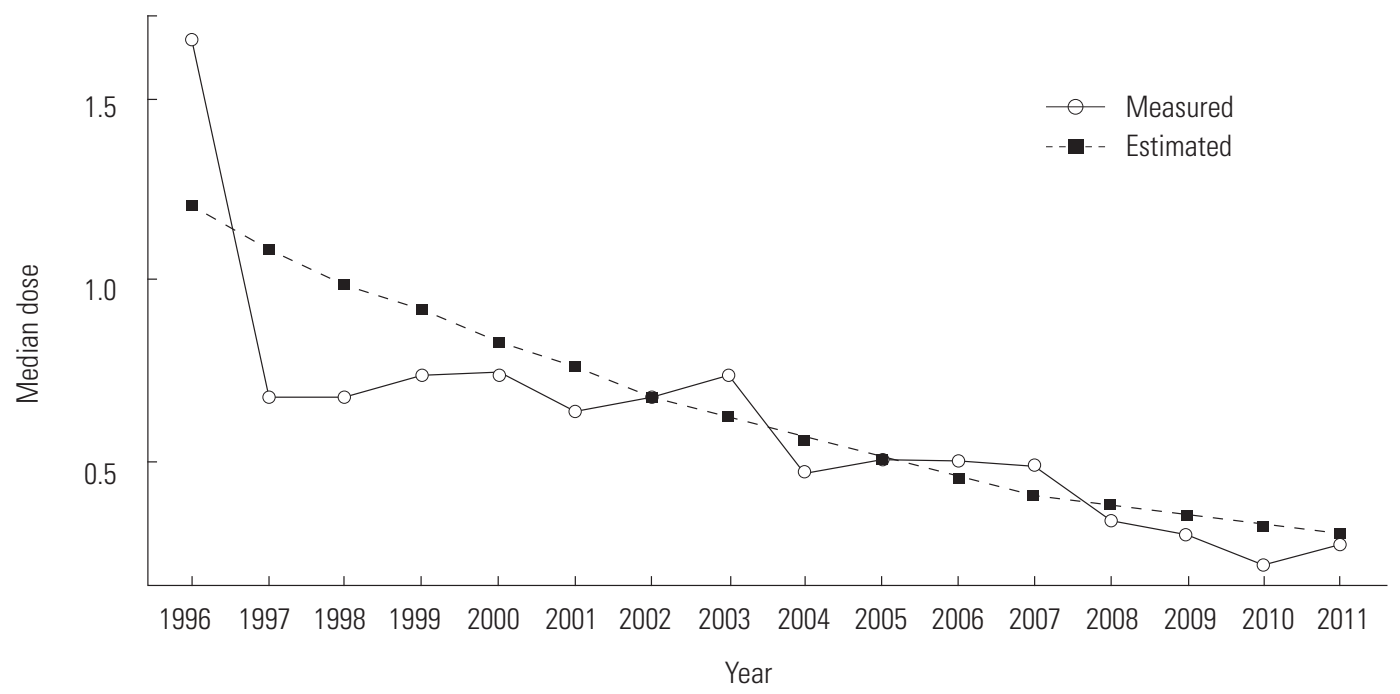

Figure 3. Comparison of median estimated doses with median measured doses for radiologic technologists, 1996-2011. 


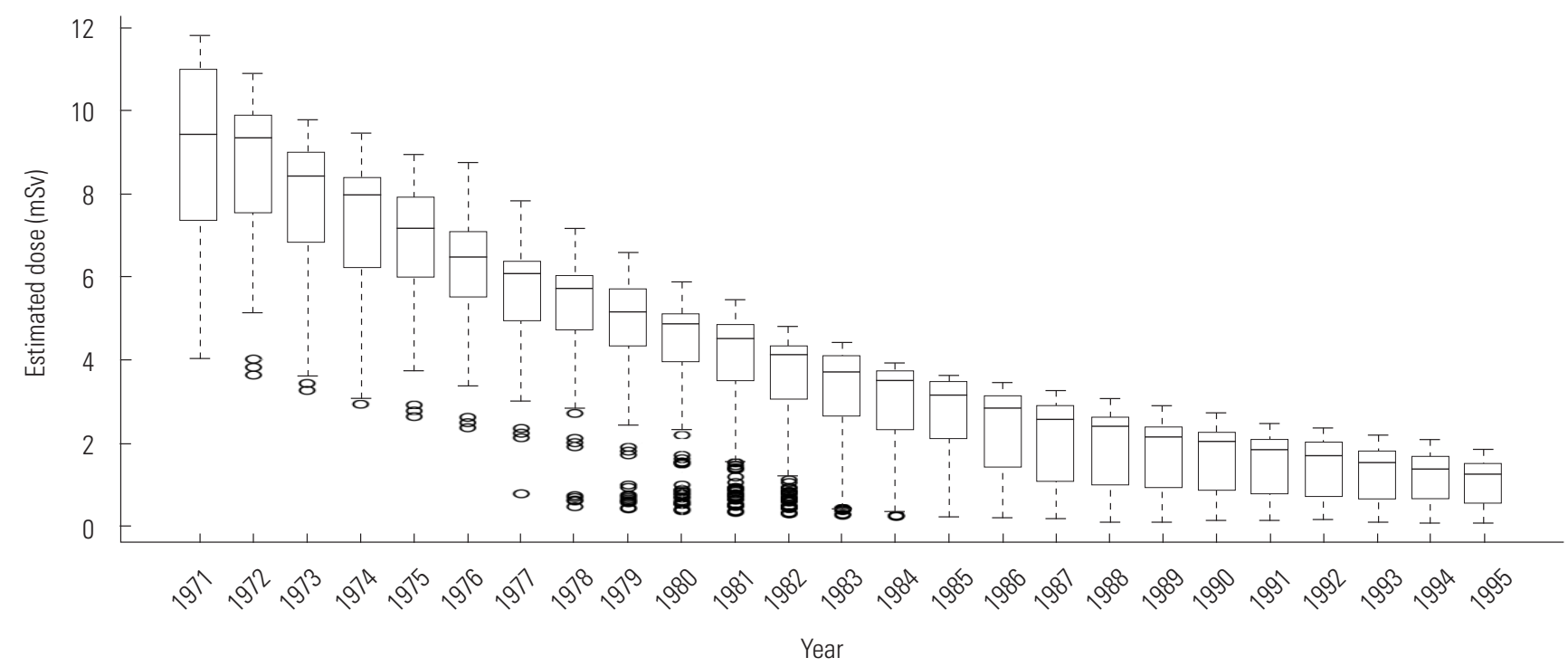

Figure 4. Distributions of annual reconstructed values for radiologic technologists, 1971-1995.

estimated in this study for the 1971 to 1995 period, for which no measured doses are available, were congruent with the results of previous historical reconstruction studies conducted on the population of medical workers in the US [6,7], China [8], and Japan [5]. Relatively large declines in earlier years were estimated, which is also consistent with the results of these previous studies. When compared with the period-specific doses estimated on the basis of the cohort of the US radiologic technologists [6,7], our estimates were relatively higher for the same time period. For example, it was estimated that during the 1977 to 1984 period, the US hospital radiologic technologists received a median dose of $2.0 \mathrm{mSv}$, while the median estimated annual doses received by their Korean counterparts during the same period of time ranged from 3.5 to $5.9 \mathrm{mSv}$. This difference might reflect both real differences (e.g., early introduction of safety regulations in the US, including the establishment of the National Council on Radiation Protection and Measurements in 1964 and the creation of the Nuclear Regulatory Commission in 1975), and differences in modeling methods (i.e., the doses in the US during the 1977 to 1984 period relied on measured badge readings during the same period, while those in this study relied entirely on data from a different period).

The reconstructed values for China [8] were more comparable with our findings. Average annual doses for four 5-yearlong periods - 1975-1979, 1980-1984, 1985-1989, and 19901994-were 6.4, 3.9, 2.8, and 2.3 mGy (1 Sv equals to 1 Gy for $\mathrm{X}$-ray radiation), respectively, for Chinese diagnostic X-ray workers, while those estimated for Korean radiologic technologists for the same time periods were 5.5, 3.4, 2.2, and 1.5 $\mathrm{mSv}$. It is also suggested that the estimates for Japanese radiologic technologists are comparable to our estimates, judging by the fact that these former estimates decreased from values slightly under 10 mSv in 1979 to slightly above 1 mSv in 1993; however, note that the estimated values were reported in a graph rather than as exact numbers [5].

As the first attempt to reconstruct past individual radiation doses among radiologic technologists in Korea, this study has some limitations that should be addressed in a future refined model. First, we applied only selected predictors for developing reconstruction models, owing to the current unfavorable paucity of information on the potential determinants of doses. It is reasonable, however, to assume that the level of exposure to radiation strongly depends on the selected predictor variables included in our model: age, sex, and the type of facility. Our results from a tree regression analysis support this. Second, the models generated in this study assumed that the type of facility at which a participant worked in 1996 to 2011 was the same at which the participant worked during the survey. This might be an unlikely assumption, considering that approximately $8.5 \%$ of radiologic technologists in Korea switch their employment every year [12]. This could lead to a misclassification of participants, subsequently distorting the estimation of parameters and dose reconstruction. Thus, knowing past work history is crucial for a more reliable estimation of radiation exposure. Third, we included only radiologic 
technologists who answered "I always wear a badge" to avoid a potential misrepresentation of true doses; however, we were unable to validate their self-reported responses. If it is assumed that a certain proportion of the eligible respondents did not actually always wear badges, then both the measured and the estimated badge doses would be systematically underestimated. Fourth, although our model satisfactorily matched the existing data, the model's predictive capability cannot be guaranteed. In this study, it was not possible to verify the reconstructed doses in the period of time preceding 1996 because pre-1996 dose data for radiologic technologists were not available. Instead, we found that the estimated doses from our model were comparable with the measured doses from the national dose registry.

In addition, this study did not consider uncertainties originating from dosimetry sensitivity and accuracy, because information on the types of badges used by the study participants and their technical specifications were not available. However, if it is assumed that sensitivity was lower and badge exchange was more frequent in the early days [13], the estimated doses in the remote past were also likely to be underestimated. A Berkson type error might also have occurred when we reconstructed the pre-1996 period doses by assigning single estimates to multiple participants within the same group, if their predictors in the model, namely measurement years and age at a specific year, were identical [14]. When reconstructed doses are used as determinant variables in epidemiologic studies, these errors should be accounted for by providing the ranges of dose estimates within which the individual doses are likely to be measured [15].

However, our models retain some strength and advantage with respect to the extensiveness and the reliability of the data used. The survey encompassed the majority (63\%) of the target population, which enabled us to incorporate radiologic technologists with a variety of demographic characteristics. Thus, a potential bias associated with participants was alleviated by the extensive inclusion of the study population. In addition, the badge readings were obtained from the national dose registry that has been collecting the data for 16 years, and these dose data provided comprehensive and reliable exposure information for our model.

In summary, we have developed a model for estimating radiation badge doses received by radiologic technologists. Although further effort should be devoted to the refinement of reconstruction methods based on more detailed information, our findings contribute to calculating cumulative occupational radiation doses, to estimating individual organ-specific doses, and to determining dose-response relationships linking radiation exposure to radiation-related health consequences.

\section{ACKNOWLEDGEMENTS}

This research was supported by a grant of the Korea Health Technology R\&D Project through the Korea Health Industry Development Institute (KHIDI), funded by the Ministry of Health and Welfare, Republic of Korea (grant no. HI16C1186).

\section{CONFLICT OF INTEREST}

The authors have no conflicts of interest associated with the material presented in this paper.

\section{ORCID}

Yeongchull Choi http://orcid.org/0000-0001-5689-6932

Jaeyoung Kim http://orcid.org/0000-0002-8604-6442

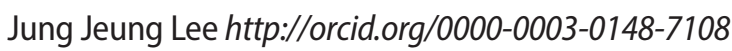

Jae Kwan Jun http://orcid.org/0000-0003-1647-0675

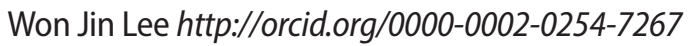

\section{REFERENCES}

1. National Council on Radiation Protection and Measurements. NCRP report no. 163: radiation dose reconstruction: principles and practices [cited 2016 Sep 4]. Available from: http://ncrp online.org/publications/reports/ncrp-report-163/.

2. Boice JD Jr, Leggett RW, Ellis ED, Wallace PW, Mumma M, Cohen SS, et al. A comprehensive dose reconstruction methodology for former rocketdyne/atomics international radiation workers. Health Phys 2006;90(5):409-430.

3. Bouville A, Toohey RE, Boice JD Jr, Beck HL, Dauer LT, Eckerman $\mathrm{KF}$, et al. Dose reconstruction for the million worker study: status and guidelines. Health Phys 2015;108(2):206-220.

4. Eheman CR, Tolbert PE. Estimating occupational radiation doses when individual dosimetry information is not available: a job exposure matrix. Am J Ind Med 1999;36(3):348-359.

5. Yoshinaga S, Yamamoto Y, Aoyama T, Yoshimoto Y. Results and problems of occupational dose reconstruction for Japanese radiological technologists. Radiat Prot Dosimetry 1998;77(12):73-78. 
6. Simon SL, Weinstock RM, Doody MM, Neton J, Wenzl T, Stewart $\mathrm{P}$, et al. Estimating historical radiation doses to a cohort of U.S. radiologic technologists. Radiat Res 2006;166(1 Pt 2):174192.

7. Simon SL, Preston DL, Linet MS, Miller JS, Sigurdson AJ, Alexander $\mathrm{BH}$, et al. Radiation organ doses received in a nationwide cohort of U.S. radiologic technologists: methods and findings. Radiat Res 2014;182(5):507-528.

8. Zhang L, Jia D, Chang H, Zhan W, Dai G, Ku M, et al. A retrospective dosimetry method for occupational dose for chinese medical diagnostic X-ray workers. Radiat Prot Dosimetry 1998;77(1-2):69-72.

9. Lee WJ, Ha M, Hwang SS, Lee KM, Jin YW, Jeong M, et al. The radiologic technologists' health study in South Korea: study design and baseline results. Int Arch Occup Environ Health 2015;88(6):759-768.

10. Lee WJ, Cha ES, Ha M, Jin YW, Hwang SS, Kong KA, et al. Occupational radiation doses among diagnostic radiation workers in South Korea, 1996-2006. Radiat Prot Dosimetry 2009; 136(1):50-55.

11. National Law Information Center. Medical Service Act; 1994 [2016 Sep 4]. Available from: http://www.law.go.kr/eng/eng LsSc.do?menuld $=1 \&$ query $=$ medical $\& \mathrm{x}=0 \& \mathrm{y}=0$ \#liBgcoor38.

12. Kim SH. An empirical study on the earnings \& labor mobility performances of qualified practitioners [dissertation]. Seoul: Sungkyunkwan University; 2010 (Korean).

13. Toohey RE. Scientific issues in radiation dose reconstruction. Health Phys 2008;95(1):26-35.

14. Armstrong BG. Effect of measurement error on epidemiological studies of environmental and occupational exposures. Occup Environ Med 1998;55(10):651-656.

15. Heid IM, Küchenhoff $H$, Miles J, Kreienbrock L, Wichmann HE. Two dimensions of measurement error: classical and Berkson error in residential radon exposure assessment. J Expo Anal Environ Epidemiol 2004;14(5):365-377. 


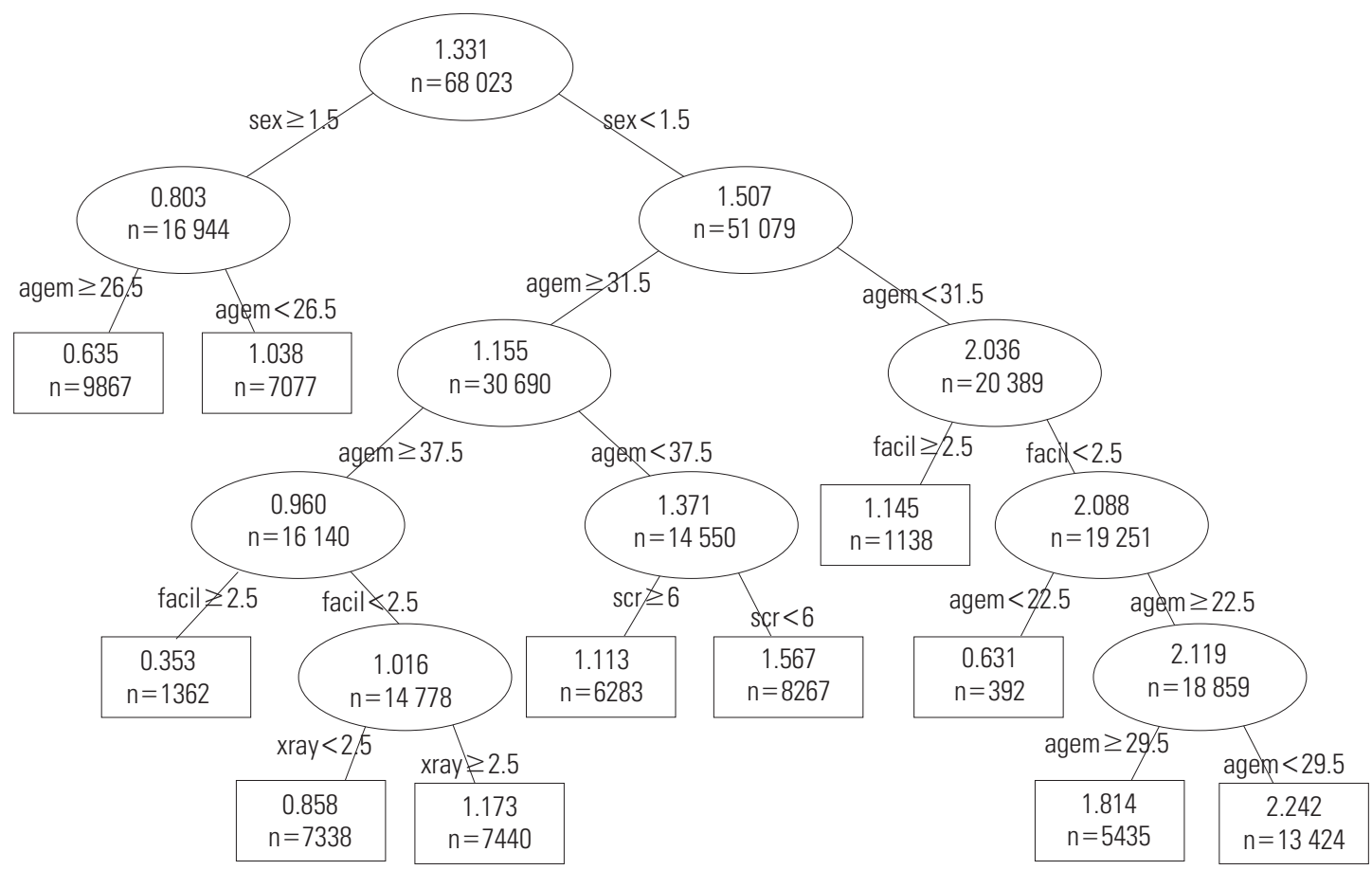

Supplemental Figure 1. Pruned regression tree of annual doses for radiologic technologists, 1996-2011. Elliptical nodes indicate internal nodes, while rectangular nodes denote terminal nodes. agem, age in the year of exposure; facil, type of facility; xray, frequency of diagnostic X-ray (categorical); scr, frequency of screen protection (categorical). 


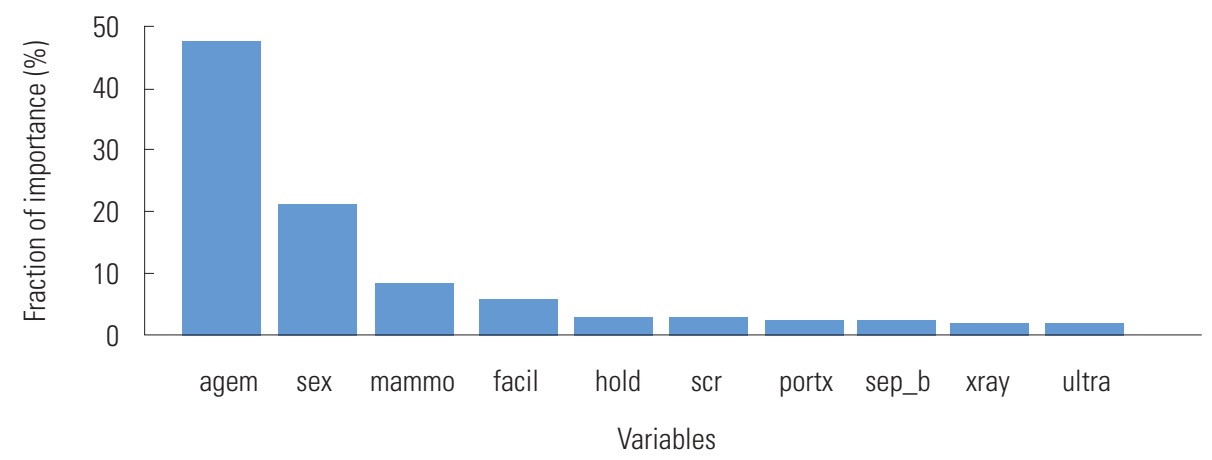

Supplemental Figure 2. Sorted variable importance in the tree regression analysis for radiologic technologists. Variable importance refers to the total amount of reduced variability (e.g., residual sum of squares) attributable to splits over each variable in a given tree. agem, age in the year of exposure; mammo, frequency of mammography; facil, type of facility; hold, whether the participant holds the patients while conducting the task or not; scr, frequency of screen protection; portx, frequency of portable X-ray; sep_b, whether the participant is always separated from the patients or not; xray, frequency of diagnostic X-ray; ultra, frequency of ultrasound examination. 


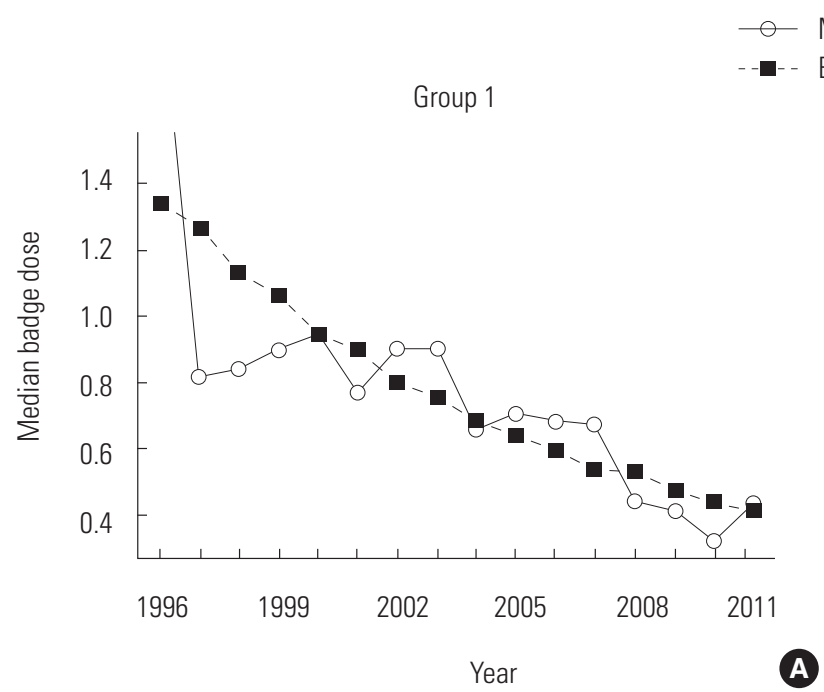

- Measured
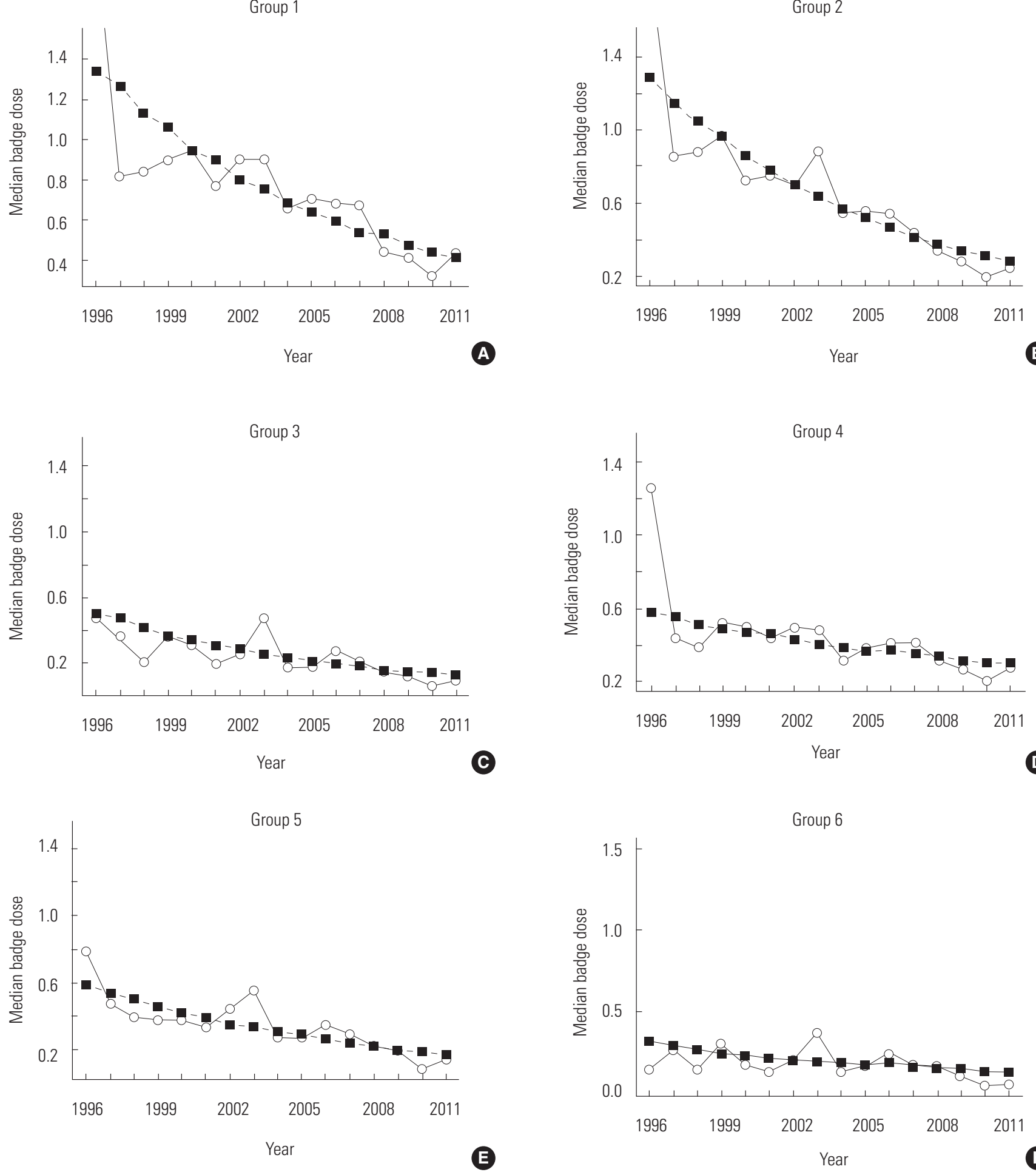

C

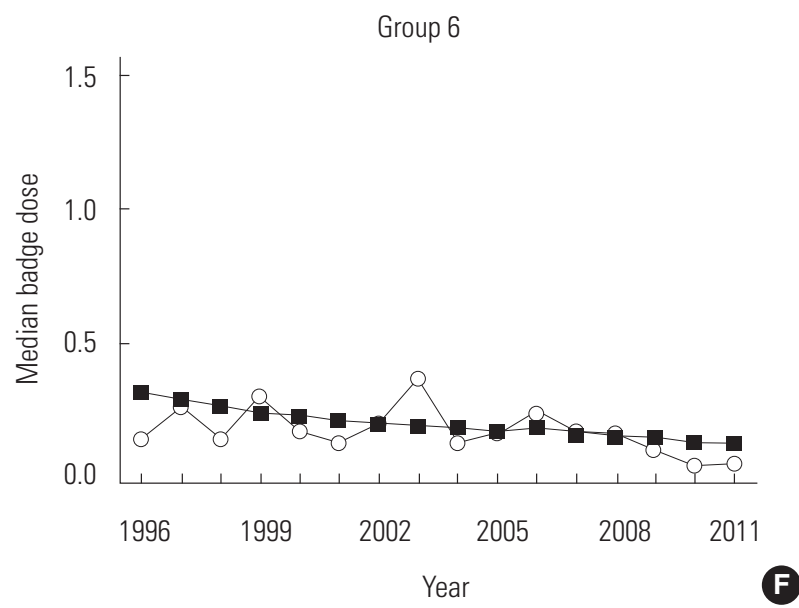

Supplemental Figure 3. Comparison of median estimated doses with median measured doses by group of radiologic technologists, 1996-2011. (A) Group 1, male hospital workers; (B) Group 2, male clinic workers; (C) Group 3, male others workers; (D) Group 4, female hospital workers; (E) Group 5, female clinic workers; (F) Group 6, female others workers. 


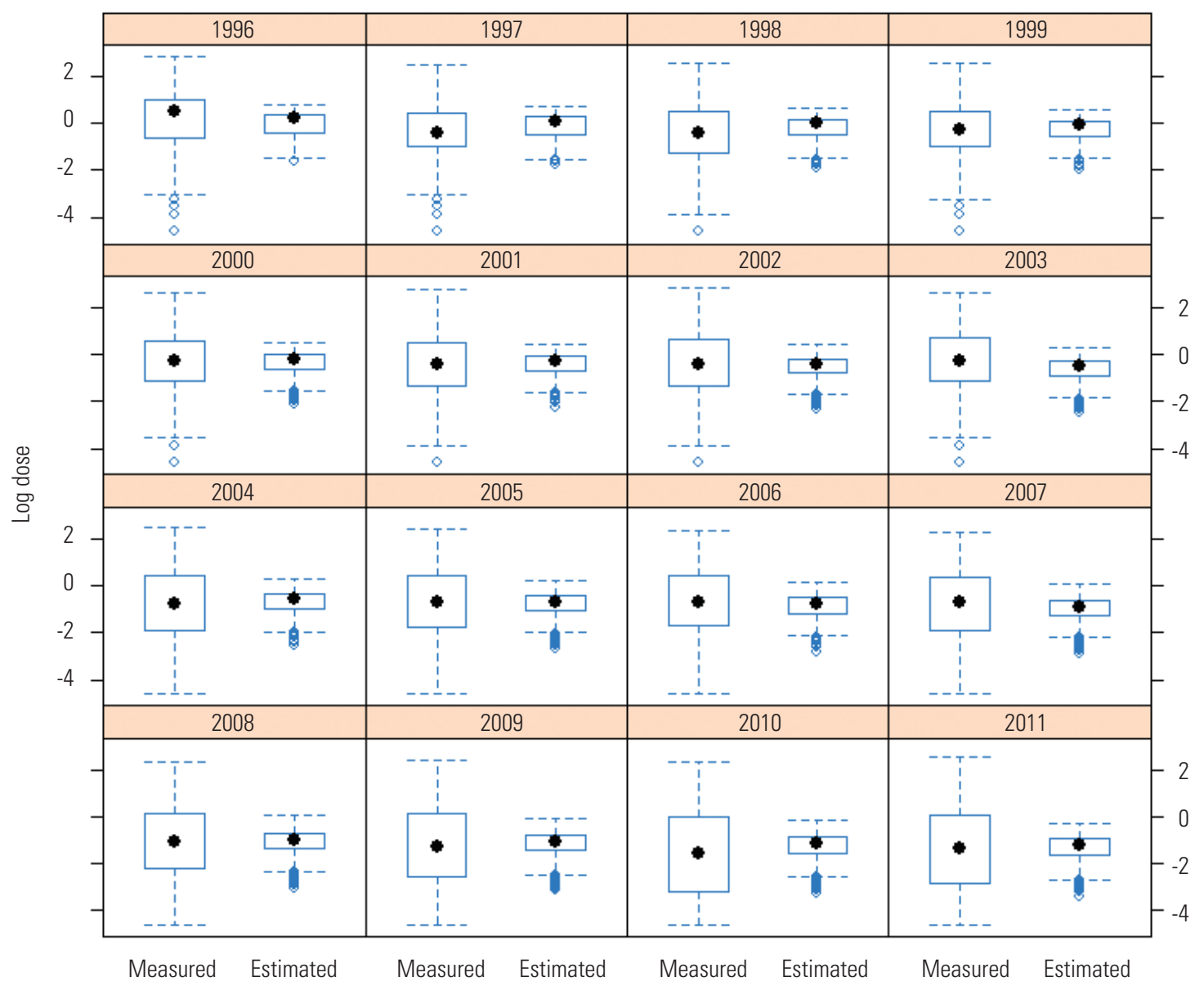

Supplemental Figure 4. Distributions of measured and estimated radiation doses for radiologic technologists, 1996-2011. 
Yeongchull Choi, et al.

Supplemental Table 1. Estimated median badge doses ( $\mathrm{mSv}$ ) in the pre-1996 period by 6 group of radiologic technologists

\begin{tabular}{|c|c|c|c|c|c|c|}
\hline $\begin{array}{l}\text { Calendar } \\
\text { year }\end{array}$ & 1 & 2 & 3 & 4 & 5 & 6 \\
\hline 1971 & 9.45 & 10.63 & - & - & - & - \\
\hline 1972 & 8.49 & 9.49 & - & - & - & - \\
\hline 1973 & 8.03 & 8.59 & - & - & - & - \\
\hline 1974 & 7.96 & 7.67 & - & - & - & - \\
\hline 1975 & 7.16 & 7.49 & - & - & - & - \\
\hline 1976 & 6.43 & 7.09 & - & - & - & - \\
\hline 1977 & 5.93 & 7.03 & 0.78 & - & - & - \\
\hline 1978 & 5.74 & 6.37 & 0.71 & 0.62 & - & 0.47 \\
\hline 1979 & 5.16 & 5.86 & 0.69 & 0.61 & - & 0.43 \\
\hline 1980 & 4.87 & 5.55 & 0.83 & 0.70 & 1.52 & 0.41 \\
\hline 1981 & 4.60 & 5.10 & 0.78 & 0.71 & 1.46 & 0.43 \\
\hline 1982 & 4.13 & 4.69 & 0.73 & 0.72 & 1.47 & 0.43 \\
\hline 1983 & 3.90 & 4.18 & 0.73 & 0.78 & 1.35 & 0.40 \\
\hline 1984 & 3.51 & 3.73 & 0.83 & 0.80 & 1.33 & 0.40 \\
\hline 1985 & 3.15 & 3.43 & 0.82 & 0.75 & 1.23 & 0.40 \\
\hline 1986 & 2.98 & 3.06 & 0.87 & 0.69 & 1.18 & 0.44 \\
\hline 1987 & 2.81 & 2.82 & 0.77 & 0.67 & 1.09 & 0.41 \\
\hline 1988 & 2.53 & 2.51 & 0.73 & 0.64 & 1.01 & 0.37 \\
\hline 1989 & 2.39 & 2.24 & 0.69 & 0.60 & 0.92 & 0.37 \\
\hline 1990 & 2.25 & 2.06 & 0.61 & 0.55 & 0.85 & 0.34 \\
\hline 1991 & 2.13 & 1.84 & 0.57 & 0.53 & 0.78 & 0.30 \\
\hline 1992 & 1.91 & 1.69 & 0.51 & 0.50 & 0.71 & 0.27 \\
\hline 1993 & 1.81 & 1.51 & 0.45 & 0.46 & 0.64 & 0.24 \\
\hline 1994 & 1.62 & 1.39 & 0.42 & 0.44 & 0.60 & 0.22 \\
\hline 1995 & 1.53 & 1.24 & 0.37 & 0.41 & 0.54 & 0.20 \\
\hline
\end{tabular}

